\title{
Transport Through a Single-Molecule Transistor: Master-Equation Approach
}

\author{
A. Donabidowicz-Kolkowska And C. Timm \\ Institut für Theoretische Physik, Technische Universität Dresden, Germany
}

\begin{abstract}
In the present work we employ the master-equation approach to describe the transport through a molecule located in the central region between two external electrodes. In contrast to the transport through a quantum dot, electron-phonon coupling should be taken into account for tunnelling through a molecule. The coupling results in the appearance of additional effects such as vibrational sidebands or, for the case of strong coupling, a suppression of the current at low bias voltage (Franck-Condon blockade). In contrast to previous studies, the transport properties are described by the density matrix calculated explicitly with diagonal and off-diagonal elements. The observed phenomena are discussed and compared to previous studies.
\end{abstract}

PACS: 73.23.Hk, 73.63.-b, 81.65.+h

\section{Introduction}

Recently, there has been increased interest in transport through single-molecule devices. Transport through molecules shows similarities to the transport through artificial quantum dots including such effects as Coulomb blockade [1, 2] and the Kondo effect [3-5]. However, the coupling of electrons on the molecule to the phonon modes [6-12] has also significant influence on the transport properties. Due to the electron-phonon coupling, vibrational sidebands can be observed as characteristic steps in the current-voltage curves. In addition, for the case of strong electron-phonon coupling, single-electron tunnelling on and off the molecule (sequential tunnelling) can be suppressed at low bias voltages. This effect is called Franck-Condon (FC) blockade.

The purpose of this article is to extend the knowledge about the transport properties of a molecular junction. In order to describe the transport through the molecule, we use a density-matrix approach including all coherent tunnelling processes [13-16], thereby avoiding the assumption of a diagonal reduced density matrix. The nonlinear transport properties are obtained from the master equation. In the present study, the limit of weak molecule-lead coupling will be employed. In this case the tunnelling Hamiltonian $H_{t}$ can be treated within perturbation theory. In the lowest order of this expansion, sequential tunnelling is obtained. Because an electron tunnelling into or out of the molecule can change the excitation of vibrational modes, off-diagonal elements of the density matrix between different excited states are taken into account.

\section{Model and method}

The system consists of a molecule coupled to a phonon mode and connected to external electrodes. It is described by the Anderson-Holstein Hamiltonian $\hat{H}=$ $\hat{H}_{\text {leads }}+\hat{H}_{t}+\hat{H}_{\text {mol }}[6,9,10]$, where the noninteracting leads are described by $\hat{H}_{\text {leads }}=\sum_{\boldsymbol{k}, \beta, \sigma} \xi_{\boldsymbol{k} \beta} \hat{c}_{\boldsymbol{k}_{\beta \sigma}}^{\dagger} \hat{c}_{\boldsymbol{k} \beta \sigma}$, the tunnelling between molecule and leads is described by

$$
\hat{H}_{t}=\sum_{\boldsymbol{k}, \beta, \sigma}\left(V_{\boldsymbol{k}_{\beta}} \hat{c}_{\boldsymbol{k}}{ }_{\beta \sigma} \hat{d}_{\sigma}^{\dagger}+\text { h.c. }\right)
$$

and

$$
\begin{aligned}
& \hat{H}_{\mathrm{mol}}=\sum_{\sigma}\left(\epsilon_{d}-e V_{g}\right) \hat{d}_{\sigma}^{\dagger} \hat{d}_{\sigma}+\frac{U}{2} \hat{n}_{d}\left(\hat{n}_{d}-1\right) \\
& \quad+\lambda \omega\left(\hat{b}^{\dagger}+\hat{b}\right) \hat{n}_{d}+\hbar \omega \hat{b}^{\dagger} \hat{b}
\end{aligned}
$$

with $\hat{n}_{d}=\hat{d}_{\uparrow}^{\dagger} \hat{d}_{\uparrow}+\hat{d}_{\downarrow}^{\dagger} \hat{d}_{\downarrow}$ contains the electronic and vibrational degrees of freedom of the molecule. Operators $\hat{c}_{\boldsymbol{k}_{\beta \sigma}}\left(\hat{c}_{\boldsymbol{k} \beta \sigma}^{\dagger}\right)$ annihilate (create) electrons in the left $(\beta=L)$ or right $(\beta=R)$ electrodes with the corresponding energies. These energies are measured from the chemical potentials shifted symmetrically by the external voltage $\mu_{L}-\mu_{R}=\mathrm{eV}$. The densities of states of the leads are approximated by constants. Operators $\hat{d}_{\sigma}$ and $\hat{d}_{\sigma}^{\dagger}$ refer to the localized electrons on the molecule with the single-particle energy $\epsilon_{d}$, which can be tuned by applying a gate voltage $V_{g}$. $U$ is the Coulomb interaction on the molecule. The tunnelling matrix elements $V_{\boldsymbol{k} \beta}$ characterize the strength of the molecule-lead coupling. Vibrational excitations with energy $\hbar \omega$ are annihilated (created) by $\hat{b}\left(\hat{b}^{\dagger}\right)$. A single vibrational mode is taken into account within the harmonic approximation. The equilibrium distances between the nuclei of the molecule are changed if an electron is added to or removed from the molecule. The coupling between vibrational and electronic degrees of freedom is described by the term $\lambda \hbar \omega\left(\hat{b}^{\dagger}+\hat{b}\right) \hat{n}_{d}$. Unlike in most previous studies, we do not apply a Lang-Firsov transformation [17] to transfer this coupling to the tunnelling term.

We introduce the reduced density matrix of the molecule as $\rho_{D}(t)=\operatorname{Tr}_{\text {leads }} \rho(t)$, where the trace is over many-particle states of the leads. We drop the subscript $D$ in the following. Using standard methods [14], the master equation for the components of $\rho$ with respect to 
the basis $\{|n, q\rangle\}$ can be written as

$$
\begin{aligned}
& \frac{d \rho_{q q^{\prime}}^{n n^{\prime}}}{d t}=-\mathrm{i}\left(E_{n q}-E_{n^{\prime} q^{\prime}}\right) \rho_{q q^{\prime}}^{n n^{\prime}}-\sum_{s q^{\prime \prime \prime}} R_{q q^{\prime \prime \prime}}^{n s} \rho_{q^{\prime \prime \prime} q^{\prime}}^{s n^{\prime}} \\
& -\sum_{l q^{\prime \prime}} R_{q^{\prime \prime} q^{\prime}}^{l n^{\prime}} \rho_{q q^{\prime \prime}}^{n l}+\sum_{s l q^{\prime \prime} q^{\prime \prime \prime}} R_{q q^{\prime \prime}, q^{\prime \prime \prime} q^{\prime}}^{1,2, n l, s n^{\prime}} \rho_{q^{\prime \prime} q^{\prime \prime \prime}}^{l s} \\
& -\left(1-\delta_{n n^{\prime}}\right) \frac{1}{\tau_{2}} \rho_{q q^{\prime}}^{n n^{\prime}} \\
& -\delta_{n n^{\prime}} \frac{1}{\tau_{1}}\left(\rho_{q q^{\prime}}^{n n}-P_{n}^{\mathrm{eq}, \mathrm{el}} \sum_{n^{\prime \prime}} \rho_{q q^{\prime}}^{n^{\prime \prime} n^{\prime \prime}}\right) .
\end{aligned}
$$

Here, $|n, q\rangle$ denotes the molecular eigenstates, where $n$ specifies the electronic state $(0, \uparrow, \downarrow$, or $\uparrow \downarrow)$, while $q$ is the harmonic-oscillator quantum number of the vibrational mode. $\tau_{1}$ and $\tau_{2}$ are off-diagonal and diagonal relaxation times, respectively, describing the effect of an additional heat bath on the electronic states. The equilibrium distribution $P_{n}^{\text {eq,el }}$ is given by the Boltzmann distribution. The transition rates $R_{q q^{\prime \prime \prime}}^{n s}$ for sequential tunnelling from state $|n, q\rangle$ to $\left|s, q^{\prime \prime \prime}\right\rangle$ contain contributions from the electronic and phononic wave functions. The electronic part is described by the couplings to the electrodes, $\Gamma_{\beta}=2 \pi \sum_{\beta} \rho_{\beta}\left|V_{\boldsymbol{k}_{\beta}}\right|^{2}$, the matrix elements of annihilation $\left(C_{n s}^{\sigma}=\left\langle n\left|\hat{d}_{\sigma}\right| s\right\rangle\right)$ and creation $\left(C_{n s}^{+\sigma}=\left\langle n\left|\hat{d}_{\sigma}^{\dagger}\right| s\right\rangle\right)$ operators and the Fermi function $f_{\beta}$ depending on the energy of the molecule in the state $|n, q\rangle$,

$$
\begin{aligned}
& E_{n q}=\left(\epsilon_{d}-e V_{g}\right) n+\frac{U}{2} n(n-1)+\left(q+\frac{1}{2}\right) \hbar \omega \\
& -n \lambda^{2} \hbar \omega .
\end{aligned}
$$

In order to simplify the form of Eq. (2), the master equation can be rewritten in the matrix form $[13,16]$

$$
\frac{\mathrm{d} \rho}{\mathrm{d} t}=M \boldsymbol{\rho},
$$

where $M$ is the $N^{2} \times N^{2}$ rate matrix and $N$ is a number of molecular state we are taking into account. In the stationary state, which satisfies $\mathrm{d} \rho / \mathrm{d} t=0$, Eq. (4) takes the form $0=M \rho$. In order to calculate the stationary occupation probabilities $P_{q q}^{s s}$, the diagonal $(d)$ and offdiagonal $(n)$ density-matrix elements can be separated into two vectors $\boldsymbol{P}_{d}$ and $\boldsymbol{P}_{n}$. Then Eq. (4) can be split into two blocks [16],

$$
\left[\begin{array}{l}
\mathbf{0}_{d} \\
\mathbf{0}_{n}
\end{array}\right]=\left[\begin{array}{cc}
M_{d d} & M_{d n} \\
M_{n d} & M_{n n}-\mathrm{i} L_{n n}
\end{array}\right]\left[\begin{array}{l}
\boldsymbol{P}_{d} \\
\boldsymbol{P}_{n}
\end{array}\right] .
$$

After solving the equation for $\boldsymbol{P}_{n}$, its solution can be inserted into the equation for $\boldsymbol{P}_{d}$. In this way the nondiagonal elements are eliminated and an effective equation $M_{d} \boldsymbol{P}_{d}=0$ for $\boldsymbol{P}_{d}$ is obtained, where

$$
M_{d}=M_{d d}^{(2)}+M_{d d}^{(4)}-\mathrm{i} M_{d n}^{(2)} L_{n n}^{-1} M_{n d}^{(2)}
$$

and

$$
L_{n n}^{-1}=\left(E_{n}^{q}-E_{n \prime}^{q \prime}\right) .
$$

This equation resembles the rate equation, but it contains the effective rates i $M_{d n}^{(2)} L_{n n}^{-1} M_{n d}^{(2)}$. By these effective rates the 4 th order effects of coherences are included.
One should emphasize that due to the absence of $M_{n n}$ the evaluation of Eq. (2) can be significantly simplified.

From the obtained reduced density matrix, one can calculate the current flowing out of the left electrode into the molecule, $I_{L}(t)=\operatorname{Tr}\left(I_{L} \boldsymbol{\rho}(t)\right)$, where $\operatorname{Tr}$ is the trace over the full system. Then, after analogous calculations for the corrections to the current one obtains $I_{r}=\boldsymbol{e}_{d}^{T}\left[M_{I_{r}}\right]_{d} \boldsymbol{P}_{d}$ with

$$
\begin{gathered}
\left(M_{I_{r}}\right)_{d}=\left(M_{I_{r}}^{(2)}\right)_{d d}+\left(M_{I_{r}}^{(4)}\right)_{d d} \\
-\mathrm{i}\left(M_{I_{r}}^{(2)}\right)_{d n} L_{n n}^{-1}\left(M_{I_{r}}^{(2)}\right)_{n d},
\end{gathered}
$$

where $\boldsymbol{e}_{d}^{T}$ is a unit vector $\boldsymbol{e}_{d}^{T}=(1, \ldots, 1)$.

\section{Results}

Using the master equation described in the previous section, we determine the current flowing through a molecule between two external electrodes. Representative results for the current as a function of the bias voltage are shown in Fig. 1 for the single-electron energy $\epsilon_{d}=0.5$ and thermal energy $k T=0.05$ in units of the phonon energy $\hbar \omega=1$. Two cases of the inter-

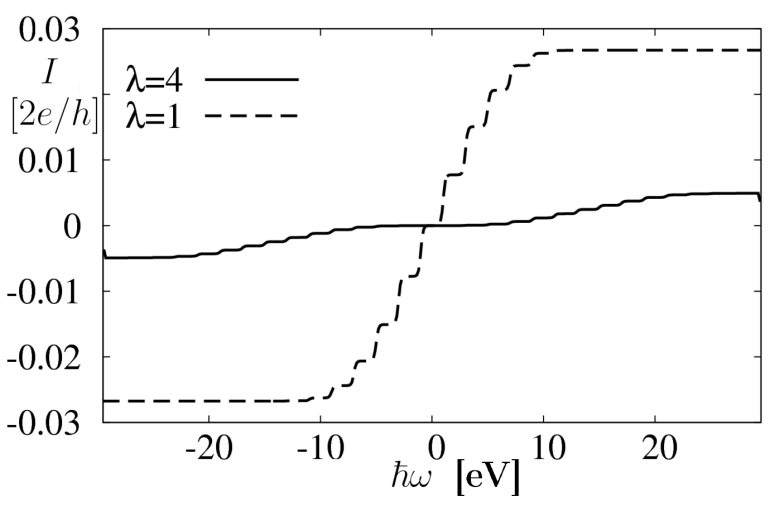

Fig. 1. Current through the molecule calculated using the full master equation for $\epsilon_{d}=0.5, k T=0.05, \hbar \omega=1$, and the two cases $\lambda=1$ and $\lambda=4$.

mediate $(\lambda=1)$ and strong $(\lambda=4)$ electron-phonon coupling are considered in the present study. For the smaller electron-phonon coupling, the current is found to be symmetric with respect to the applied source-drain voltage $V$. Moreover, it increases with the bias voltage with characteristic steps in the $I V$ characteristic. The observed steps correspond to transitions between molecular states. Additional steps observed for higher bias voltages appear due to vibrational states of the molecule. Each subsequent step is observed when $\mathrm{eV}$ is increased by twice an integer multiple of the phonon frequency. For strong electron-phonon coupling another characteristic feature is observed: The low-bias current is suppressed due to the Franck-Condon blockade [11, 13]. The distribution of the transition rates broadens considerably, opening a gap of suppressed transitions between lowlying phonon states due to very small FC matrix elements $F_{q q^{\prime}}$. Figure 2 shows the corresponding conduc- 


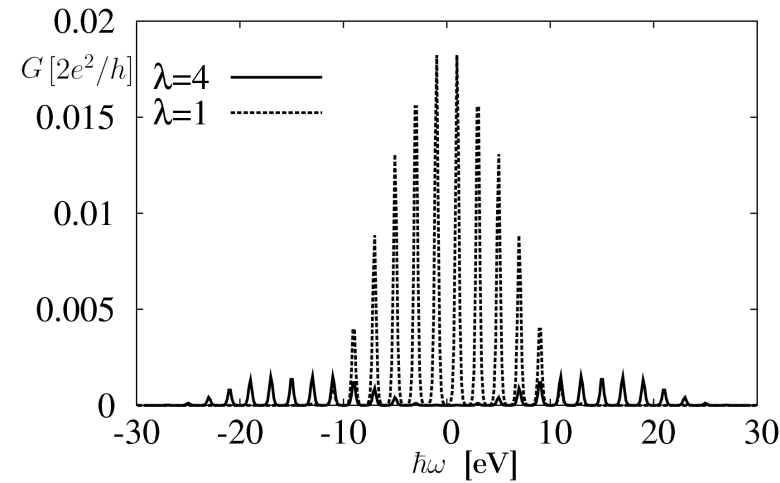

Fig. 2. Conductance through the molecule calculated using the full master equation for the same parameters as in Fig. 1.

tance $\mathrm{d} I / \mathrm{d}(\mathrm{eV})$ as a function of applied bias voltage. The weights of the conductance peaks observed in Fig. 2 are determined by the FC factors.

Similar results to those shown in Figs. 1 and 2 have been reported in Ref. [11], where off-diagonal components of the reduced density operator were ignored. Evidently, taking these off-diagonal components into account does not qualitatively change the $I V$ characteristics. Further studies, possibly taking higher-order processes into account, are necessary to clarify this result. We note that our results are in good qualitative agreement with experimental data reported by different groups [3, 18]. FC blockade has also been observed in the differential conductance of quantum dots formed in suspended singlewall carbon nanotubes [18], where the electron-phonon coupling is strong. Moreover, the temperature dependence of the experimental data confirms that the sidebands originate from excited vibronic states.

\section{Summary and conclusions}

Using a master-equation approach, we have investigated electron transport through a single molecule. In particular, we have included off-diagonal matrix elements of the reduced density matrix. In good agreement with previous studies [11, 13], the strong suppression of the current in case of strong electron phonon coupling, independently of the local Coulomb interaction $U$, was observed. The effect of including the off-diagional components is found to be small.

\section{Acknowledgements}

We gratefully acknowledge financial support by the Deutsche Forschungsgemeinschaft.

\section{References}

[1] J. Park, A.N. Pasupathy, J.I. Goldsmith, A.V. Soldatov, C. Chang, Y. Yaish, J.P. Sethna, H.D. Abruna, D.C. Ralph, P.L. McEuen, Thin Solid Films 438439, 457 (2003).

[2] L.H. Yu, D. Natelson, Nano Lett. 4, 79 (2004).

[3] J. Park, A.N. Pasupathy, J.I. Goldsmith, C. Chang, Y. Yaish, J.R. Petta, M. Rinkoski, J.P. Sethna, H.D. Abruna, P.L. McEuen, D.C. Ralph, Nature 417, 772 (2002).

[4] W. Liang, M.P. Shores, M. Bockrath, J.R. Long, H. Park, Nature 417, 725 (2002).

[5] J. Paaske, K. Flensberg, Phys. Rev. Lett. 94, 176801 (2005).

[6] L.I. Glazman, R.I. Shekhter, Zh. Eksp. Teor. Fiz. 94, 292 (1987).

[7] D. Boese, H. Schoeller, Europhys. Lett. 54, 668 (2001).

[8] M. Galperin, M.A. Ratner, A. Nitzan, Nano Lett. 4, 1605 (2004); M. Galperin et al., J. Chem. Phys. 121, 11965 (2004).

[9] A. Mitra, I. Aleiner, A.J. Millis, Phys. Rev. B. 69, 245302 (2004).

[10] S. Braig, K. Flensberg, Phys. Rev. B. 68, 205324 (2003); S. Braig, K. Flensberg, Phys. Rev. B. 70, 085317 (2004).

[11] J. Koch, F. von Oppen, Phys. Rev. Lett. 94, 206804 (2005); J. Koch, M.E. Raikh, F. von Oppen, Phys. Rev. Lett. 95, 056801 (2005).

[12] F. Elste, G. Weick, C. Timm, F. von Oppen, Appl. Phys. A 93, 345 (2008).

[13] M. Leijnse, M.R. Wegewijs, Phys. Rev. B. 78, 235424 (2008).

[14] C. Timm, Phys. Rev. B. 77, 195416 (2008).

[15] M.G. Schultz, F. von Oppen, Phys. Rev. B. 80, 033302 (2009).

[16] S. Koller, M. Grifoni, M. Leijnse, M. R. Wegewijs, Phys. Rev. B. 82, 235307 (2010).

[17] I.G. Lang, Y.A. Firsov, Zh. Eksp. Teor. Fiz. 43, 1843 (1962).

[18] R. Luturcq, C. Stampfer, K. Inderbitzin, L. Durrer, C. Hierold, E. Mariani, M.G. Schultz, F. von Oppen, K. Ensslin, Nature Phys. 5, 317 (2009). 